\title{
Characterization of broom fibers for PRB in the remediation of aquifers contaminated by heavy metals
}

\author{
C. Fallico, S. Troisi, A. Molinari, and M. F. Rivera \\ University of Calabria, Department of Soil Conservation, Rende (CS), Italy \\ Received: 11 January 2010 - Published in Biogeosciences Discuss.: 25 February 2010 \\ Revised: 25 June 2010 - Accepted: 3 August 2010 - Published: 25 August 2010
}

\begin{abstract}
The present level of pollution, increasingly involving ground waters, constitutes a serious risk to the environment and also to human health. Therefore the remediation of saturated and unsaturated soils to remove pollutant materials is more and more frequently required. In the present paper, the possibility of removing heavy metals by permeable reactive barrier (PRB) from the groundwater carried out specifically with broom fibers, is investigated.

Once shown the economic benefits deriving from the use of this plant, a hydraulic characterization of the broom fiber mass was performed, determining the permeability and the porosity in correspondence to different levels of compactness of the fibers.

Having verified the effectiveness of removal of some heavy metals by these fibers, the results of some experiments, carried out in the laboratory for this purpose, are shown. These experiments were carried out utilizing broom fibers obtained in different ways and, limitedly to the considered pollutants, showed the high capability of these fibers to reduce their concentrations. The best results were obtained for the broom fibers extracted by a particular chemical-physical process.

Moreover, the behaviour of this fiber with time was investigated, determining the kinetic constant of degradation.
\end{abstract}

\section{Introduction}

The pollution of the environment is a consequence of human activities, both civil and industrial and for this reason is widespread. The phenomenon involves soil, groundwater, shallow water and fresh and saltwater. The impact of this pollution is often very great especially in terms of human health,

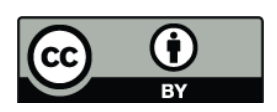

Correspondence to: C. Fallico

(fallico@dds.unical.it) in the balance of ecosystems, habitat destruction, effects on the food chain, landscape, soil used for agricultural purposes and last, but not least, on biodiversity.

The problem of soil and groundwater contamination during the past decades, owing to a progressive increase of industrial activities, is becoming increasingly alarming, both in terms of extension and in the concentration of contaminated areas. Considering these problems, the remediation of contaminated soils and aquifers is a useful mechanism for the recovery and revalorization of various areas that otherwise would be lost or that would be degraded over time.

Regarding the remediation of aquifers, there are numerous existing techniques in the literature, each of them determines a higher or lower level of efficiency in terms of removing the contaminant. In particular, the technique to be used should be chosen case by case in relation to the characteristics of the aquifer and the type of contaminant which needs to be removed. Among the various remediation techniques, in this study we refer to the use of PRB (Permeable Reactive Barrier), which is a passive technique for in situ remediation.

The permeable reactive barrier (PRB) is an effective alternative to conventional remediation methods (such as pumpand-treat, physical-chemical, etc.) for groundwater remediation. It has gained popularity, because of its efficient removal of pollutants and low operating and maintenance costs (Jun et al., 2008).

The concept of PRBs is relatively simple: reactive material is placed in the subsurface to intercept a contaminated plume that moves through it under natural gradient (Zolla et al., 2007). As the groundwater passes through the reactive media, the occurring physical and chemical processes transform the contaminants to less harmful or immobile species (Gillham et al., 1994; Orth et al., 1996, 1998). In particular, the permeable reactive barrier (PRB) instead of limiting the migration of the contaminant plume, in the way barriers do to the groundwater, provides a preferential direction where the contaminant, moved due to the natural gradient of the aquifer

Published by Copernicus Publications on behalf of the European Geosciences Union. 


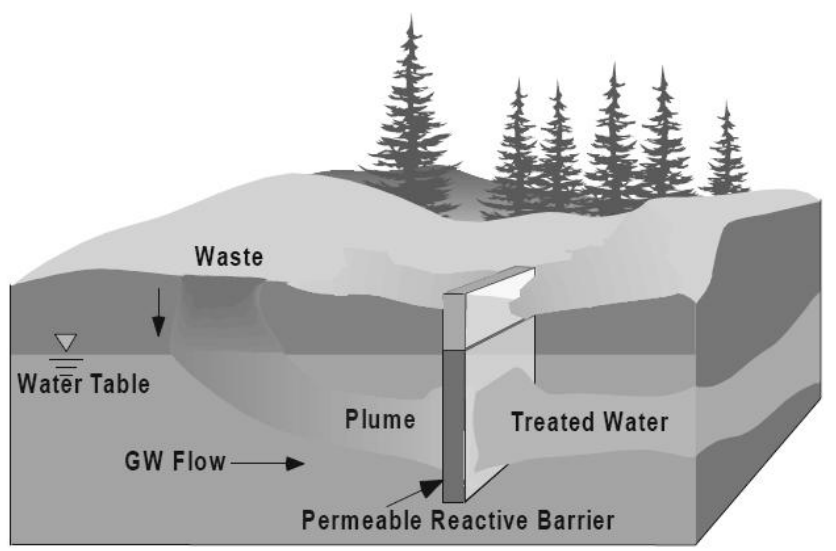

Fig. 1. Schematic of a permeable reactive barrier (PRB) (Powell et al., 1998).

(in situ passive system), is treated by the reactive means inserted and then exits from the barrier at zero concentration or in compliance with the legal limits (Fig. 1).

There are many reactive materials that can be used to fill in a permeable barrier and to make the most appropriate choice, various parameters should be taken into account.

This will depend on the reactive material characteristics and their behavior with respect to contaminants to be removed. Certainly the fundamental parameter for a good choice of reactive material is the area of the specific surface, which must be maximized to facilitate oxide reduction processes that develop the internal barrier. In any case, special attention must be given to the value of hydraulic conductivity that is created in the cell filled with the reactive species chosen. In fact, if the hydraulic conductivity in the barrier is lower than that of the surrounding aquifer, the plume may change its natural pathway, bypassing the barrier and veering towards where the hydraulic conductivity is greater, as shown in Fig. 2. Moreover, very often the cost of the various reagent materials cited in the literature, which can be considered for the realization of a PRB (Di Molfetta and Sethi, $2000,2001)$, is high or their availability is difficult, so there is a continuous research for alternative means at low cost and at greater and easier availability.

Among the materials that can be used to fill a PRB, granular zero-valent iron $\left(\mathrm{Fe}^{0}\right)$ is the most widely used compound owing to its easy availability and its affordability.

Among the innovative materials, ones which present potentially high specific area and a high porosity, are natural fibers and in particular, broom fibers.

\subsection{Natural fibers}

Natural fibers have very important characteristics for the purposes considered here. Referring to vegetal fibers (e.g. cotton, flax, hemp, jute), they are composed of cellulose and can
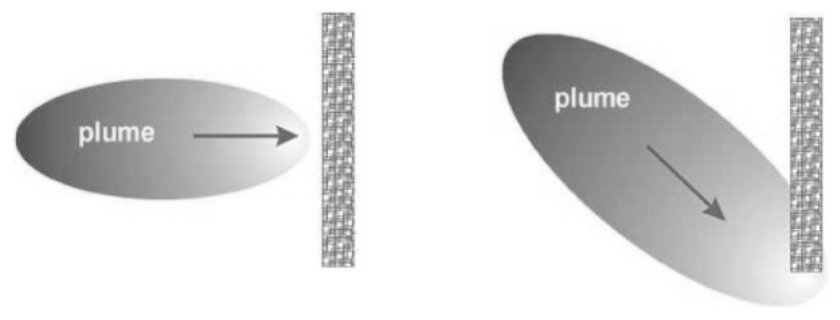

Fig. 2. PRB bypass phenomenon (Bonomo, 2003).

be generally classified as liberian, leaf or semi-fiber, depending on their origin.

Properties such as density, electrical resistivity, tensile strength, initial modulus, etc., are linked to the internal structure and chemical composition of the fibers. Natural fibers vary greatly in diameter along the length of individual filaments. The quality, as well as most of the other properties, depends on factors such as size, maturity, as well as processing methods adopted for the extraction of fiber (Mohanty et al., 2000).

A very important element that characterizes most fibers is lignin, which is a natural polymer whose structure is very complex, three-dimensional and with many ramifications of hydroxyl groups. Although it has a relatively low molecular weight, lignin is not soluble in water, has a strong resistance to chemical reactions and a high surface area $\left(180 \mathrm{~m}^{2} / \mathrm{g}\right)$. The molecular weight of lignin varies from 2000 to $15000 \mathrm{~g} / \mathrm{mol}$. These properties of lignin reveal that it has good potential to be used as a means of absorption for removing heavy metals from wastewater (Mohanty et al., 2000). However, the individual properties of the filaments and fibers may vary considerably depending on the source, age, separation technique, moisture content, test speed, history of the fiber, etc.

Studies concerning the interaction between heavy metals and natural fibers showed that the lignin in the fibers play an important role in the process of adsorption of metal cations. The results from Beom-Goo Lee (Lee et al., 2004) are very significant. This study shows that lignin plays a fundamental role in the process of adsorption of heavy metals. It remains the main mechanism determining a reduction of heavy metals through the broom fibers, but at same time it is not directly correlated to the content of lignin, because the adsorption process depends on many factors, including the concentration of metallic cations in solution, i.e. the quantity of reactive fiber, or the contact time between the solution and the fiber.

\subsection{Broom fibers}

Among the various kinds of natural fibers, those obtained from Spanish Broom (Spartium junceum) are specially interesting. It is a plant-type shrub widespread in the natural state on slopes and difficult, dry, sandy or rocky soil well-exposed to the sun. 
Its branches are called shoots with a green-glaucous color and few small deciduous leaves. The yellow flowers appear at the end of its branches from May to July. Broom is a plant characteristic of hot temperate zones, with mild and humid winters. It is naturally present in the entire Mediterranean basin, from southern France to Asia Minor and is also widely diffused on the Atlantic coast of Morocco, Portugal and in the Canary Islands.

Broom fiber is constituted of elementary fibers with a diameter of between 5 and $10 \mu \mathrm{m}$, connected with each other by lignin fibrous bundles with a diameter of about $50 \mu \mathrm{m}$ (Angelini et al., 2000). Elementary fibers are generally between 5 and $20 \mathrm{~mm}$ long, while the beam has fibrous lengths between 75 and $90 \mathrm{~cm}$, and a specific weight of $1.45 \mathrm{~g} / \mathrm{cm}^{3}$. The elementary broom fiber is comparable in length and diameter to elementary fibers of hemp, flax and cotton grown in India. It is shorter than the other types of cotton and longer and thinner than jute. The analysis of the composition of this fiber shows a high cellulose content, above $70 \%$ and a total lignin content of $6.6 \%$. The mechanical characterization of the fiber bundles have a tensile strength that varies between 400 and $700 \mathrm{MPa}$ and the tensile modulus is about $21 \mathrm{GPa}$. These values can be better understood when compared to the mechanical properties of glass fiber (tensile strength $\sim 2000 \div 3000 \mathrm{MPa}$, elastic modulus $\sim 72 \mathrm{GPa}$ ) and polymers (tensile strength $\sim 20 \div 80 \mathrm{MPa}$, modulus $\sim 1 \div 3 \mathrm{GPa}$ ). The resistance of the broom fiber increases with moisture, also when subjected to prolonged immersion in water it does not alter or lose its characteristics. The alkalis, even at high concentrations, do not affect the fiber which on the contrary are very sensitive to the action of acids.

There are numerous broom fiber applications. The present study evaluates the feasibility of using drains made from broom fiber, for the remediation of contaminated aquifers. More specifically this refers to the technology of the PRB, both active and passive, consisting in bio-drainage in natural fiber with a high surface area to be used in the remediation of groundwater contamination. Bio-drainage can be used both in passive PRB that intercepts the contaminant without changing its pathway and in active PRB that, through a water flow induced by groundwater pumping, forces the contaminant to pass through the reactive means (Troisi et al., 2008). This situation can be particularly convenient if the contaminated site which needs to be cleaned shows hot-spots or areas where the local concentrations are very high compared to the rest of the site. In fact, in such cases a cost-effective remediation would be to put an active annular PRB in the center of the hot-spot together with a well with pumping to induce a radial water movement from the aquifer towards the well itself. Here the contaminant forced to cross the barrier could be captured by reactive means. The purified water from the active annular PRB, within the limits of the law of the state in which it operates, may be discharged into any receiving water body. If those limits were not respected it could im-

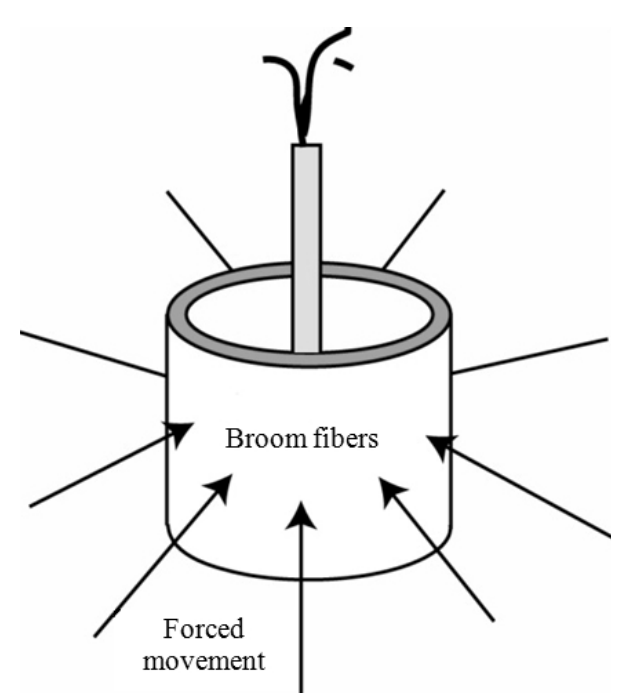

Fig. 3. Outline of annular PRB operation.

pose a recycling, returning it upstream, outside the barrier's cylinder so that it can filter again through the annular PRB. Figure 3 shows the outline of an active annular PRB with bio-drainage.

This technique, commonly used to remediate metalcontaminated areas, is able to reduce their toxicity or to remove the cations from the soil or from contaminated water.

Following this issue in the present paper some characteristics of natural fibers were evaluated, particularly broom fibers, in order to determine their effectiveness in removing a specific type of pollutant constituted of heavy metals and to be able to provide a possible alternative reagent use as a tool in the remediation of soil through PRB.

Heavy metals have high values of atomic mass and density and concentration values exceeding certain limits; moreover, they can be highly toxic and dangerous to human health. This is true especially in mining and abandoned industrial areas where the risk of contamination by these substances is high, because they are uncontrolled, since the activity of controlling the spreading of the pollutant is no longer operational or over time its effectiveness has been reduced. Considering these problems a series of laboratory tests were carried out which show the effectiveness of depuration by the broom fibers of some heavy metals in relation to different modes of use.

\section{Laboratory investigations}

Based on information obtained from the literature, that broom fiber contains lignin, that this somehow leads to an adsorption of heavy metals, and that it has good mechanical properties which make it possible to use in the construction of drains, a series of laboratory tests were conducted to verify its efficiency in purifying water containing heavy metals. 


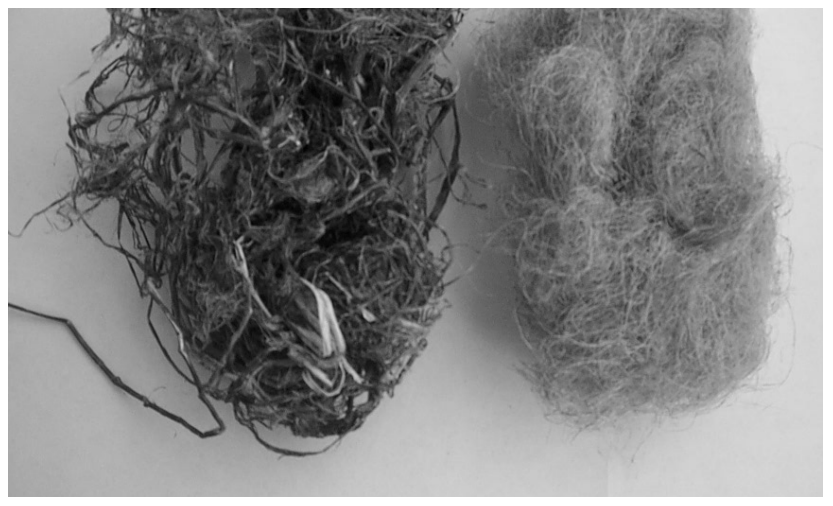

Fig. 4. Broom shoots (left); raw fibers extracted (right).

In particular, a series of tests were carried out in batch and column as well as a characterization of the fiber for the determination of hydraulic conductivity.

The fibers used in the tests were obtained manually from broom shoots, mainly from the branches of the plant, previously washed and dried and then separated from the woody part to obtain a semi-rough fiber. In Fig. 4 the shoots (washed and dried) and the fibers extracted from them are shown.

Some experiments were also performed on extracted fibers from broom through a physical-chemical process called "DiCoDe" (digestion-compression-decompression) (Chidichimo et al., 2006; Gabriele et al., 2009), using autoclave treatment of raw fibers to derive more refined and softer fibers.

There are also other fiber extraction processes, including chemical ones. These, however, can change some properties of the structure of the fiber, affecting the adsorption capacity.

\subsection{Hydraulic characterization test of broom fibers}

Broom fibers were characterized by experimental tests to determine the hydraulic conductivity or permeability and the porosity.

To evaluate empirically the permeability coefficient, laboratory tests were performed at constant head specifically on the raw fibers, even if some tests were carried out also on the DiCoDe fibers. The device used for the tests consists of a flow cell, which contained broom fibers which needed to be characterized, suitably connected by rubber pipe to a Marriott's bottle, which has a plastic tube able to scroll through the closure top up or down, allowing the adjustment of the hydraulic head for different tests. The cell, shown in Fig. 5, is connected in such a way that the water flow is directed from bottom to top of the column to permit the escape of any air bubbles. It was also possible to identify relationships that enable the estimation of permeability as a function of density, that is of the compaction degree of the fibers. Specifically that was carried out for two values of hydraulic head $(H)$ equal to $25 \mathrm{~cm}$ and $50 \mathrm{~cm}$.

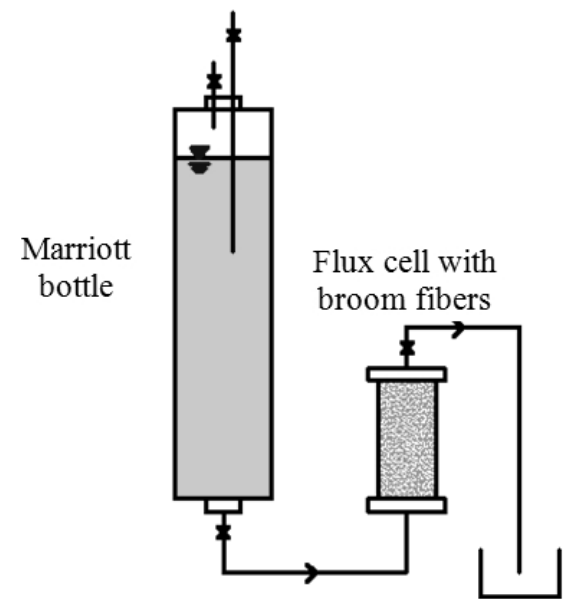

Fig. 5. Scheme of device used to measure the permeability.

\subsection{Batch tests}

Through batch tests a broad interpretation of the interaction between the broom fiber and contaminants was sought. In particular, an attempt was made to clarify the adsorption capacity with regard to the metal cations cadmium, zinc and lead in aqueous solution.

A certain amount of semi-rough broom fiber was placed in a glass container in contact with a $100 \mathrm{cc}$ aqueous solution, with a known concentration of heavy metals. The system-fiber solution, maintained at constant temperature, was stirred at intervals on a more or less regular basis so that all the solution might come into contact with the fiber. After the established contact time, the solution was analyzed and the difference between the initial and final metal concentrations provided a measure of adsorption to the fiber of the cations considered.

To take into account how the concentration $C$, the amount of fiber $P$ and the reaction time $T$ affect the adsorption process, the tests were conducted by varying one parameter at a time.

In particular were considered

- two contact times: $T_{1}=24 \mathrm{~h}, T_{2}=48 \mathrm{~h}$;

- two quantities of fiber: $P_{1}=1 \mathrm{~g}, P_{2}=2 \mathrm{~g}$;

- two sets of metal concentrations: $C_{1} \rightarrow \mathrm{Cd}=50 \mu \mathrm{g} / \mathrm{l}$, $\mathrm{Pb}=100 \mu \mathrm{g} / \mathrm{l}, \quad \mathrm{Zn}=30000 \mu \mathrm{g} / \mathrm{l}, \quad C_{2} \rightarrow \mathrm{Cd}=100 \mu \mathrm{g} / \mathrm{l}$, $\mathrm{Pb}=200 \mu \mathrm{g} / \mathrm{l}, \mathrm{Zn}=60000 \mu \mathrm{g} / \mathrm{l}$.

Then, a set of concentrations $C_{1}$, amount of fiber $P_{1}$ were set, to test the influence of contact time at $T_{1}$ and $T_{2}$.

Furthermore, to assess how a chemical treatment may influence the degree of adsorption between the fiber and heavy metals, one gram of fiber obtained by treating the shoot with a $1 \%$ solution of sodium hydroxide $(\mathrm{NaOH})$ was tested in batches at $T_{1}$ and $T_{2}$. 


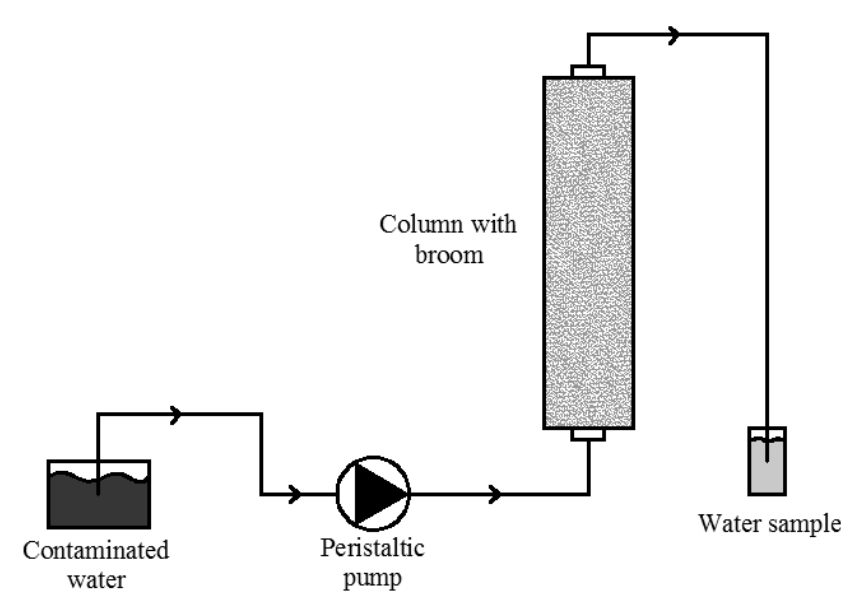

Fig. 6. Schematic representation of the experimental device used in the column test.

From the comparison between these tests and those carried out on equal terms of non-treated fiber it can be seen how treatment with $\mathrm{NaOH}$ could have influenced the adsorption process.

\subsection{Column tests}

The column tests are laboratory tests, conducted continuously, which determine the process parameters under dynamic conditions of flow and therefore in conditions closer to real ones. This is the biggest advantage of the column test compared to the batch test, which is discontinuous, less costly and challenging, but at the same time even less realistic.

The column test was used to characterize the hydraulic drainage broom fiber which is the reagent means used for testing. Thus it is indeed possible to verify that the structure, texture, location and degree of compaction of the fiber in the column is such as to determine a system of speed that is plausibly close to the normal values of water flow underground. At the same time it is important that the velocity value chosen is still capable of ensuring an adequate contact time between the solution and the medium contaminated reagent, that is the broom fiber, in order to ensure a complete process of detention and degradation of the contaminant. Again, the interpretation of column test lets us find the kinetic constant of degradation $(\lambda)$ of the contaminant. This parameter is of fundamental importance for the design of a reactive barrier, because from this value the residence time of the contaminant within the barrier can be defined to obtain the degree of reduction desired, the amount of reagent to be used and the thickness of the barrier along the direction of groundwater flow.

All tests were carried out in a column by setting the peristaltic pump at $5 \mathrm{r} / \mathrm{min}(\sim 10 \mathrm{~m} /$ day $)$ in a way that still have a speed value plausibly close to the normal rate of filtration in the groundwater.

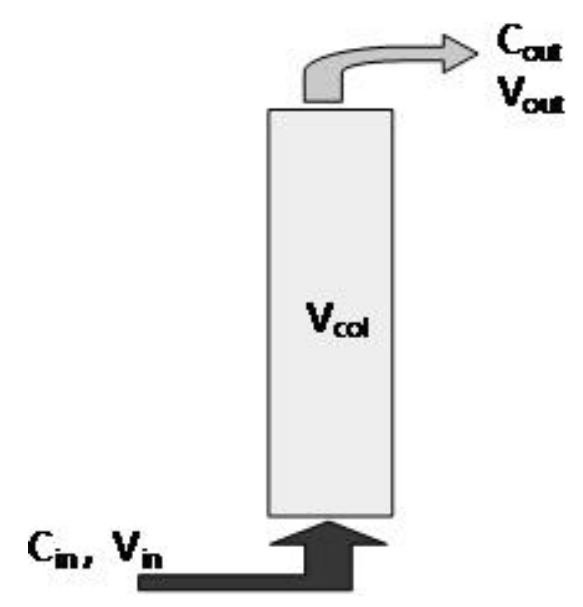

Fig. 7. Scheme of incoming and outgoing flow from the measurement column.

For the tests solutions were used based on: $\mathrm{Cd}, \mathrm{Cu}, \mathrm{Pb}$, $\mathrm{Zn}$. Because of the higher frequency of the presence of nitrate ions in soils, it was decided to use the nitrates of these metals: Cadmium nitrate tetra-hydrate $\left[\mathrm{Cd}\left(\mathrm{NO}_{3}\right)_{2} \cdot 4 \mathrm{H}_{2} \mathrm{O}\right]$, copper nitrate tri-hydrate $\left[\mathrm{Cu}\left(\mathrm{NO}_{3}\right)_{2} \cdot 3 \mathrm{H}_{2} \mathrm{O}\right]$, lead nitrate $[\mathrm{Pb}$ $\left.\left(\mathrm{NO}_{3}\right)_{2}\right]$, zinc nitrate hexa-hydrate $\left[\mathrm{Zn}\left(\mathrm{NO}_{3}\right)_{2} \cdot 6 \mathrm{H}_{2} \mathrm{O}\right]$.

The threshold limit values of contaminants (TLVs) from Italian regulation (Ministero dell'Ambiente, 2006) are:

Cadmium, $\mathrm{Cd} \rightarrow 5 \mu \mathrm{g} / \mathrm{l}=5 \times 10^{-6} \mathrm{~g} / \mathrm{l}$

Copper, $\mathrm{Cu} \rightarrow 1000 \mu \mathrm{g} / \mathrm{l}=10^{-3} \mathrm{~g} / \mathrm{l}$

Lead, $\mathrm{Pb} \rightarrow 10 \mu \mathrm{g} / \mathrm{l}=10^{-5} \mathrm{~g} / \mathrm{l}$

Zinc, $\mathrm{Zn} \rightarrow 3000 \mu \mathrm{g} / \mathrm{l}=3 \times 10^{-3} \mathrm{~g} / \mathrm{l}$

It was chosen to implement the solutions with concentrations equal to 10 times the TLVs.

The tests were conducted with the apparatus sketched in Fig. 6. This consisted in pouring through a $60 \mathrm{~cm}$ long Plexiglas column with internal diameter of $4.4 \mathrm{~cm}$ and filled with broom, a well-known solution of heavy metal pollutant concentration and volume (1.51) and measuring their concentrations at the output of the column.

The flow of contaminants through the column was set from the bottom to top by allowing the escape of any air bubbles, thus avoiding the obstructions that may be created with the formation of preferential flow paths. Instead, being directed from below the flow gradually pushes upwards by the law of Henry, allowing the air bubbles to escape from the column without causing flow problems. Considering the concentration values obtained, appropriate.

Assessments were performed periodically, including the estimation of the degree of pollutant removal by the fiber. The test was carried out both with raw fiber and fiber subjected to the DiCoDe treatment, using the same procedures, except that for the latter we conducted a temporal sampling at the exit from the column filtering.

Another parameter that can help in evaluating the effectiveness of treatment is the rate of removal of contaminants 


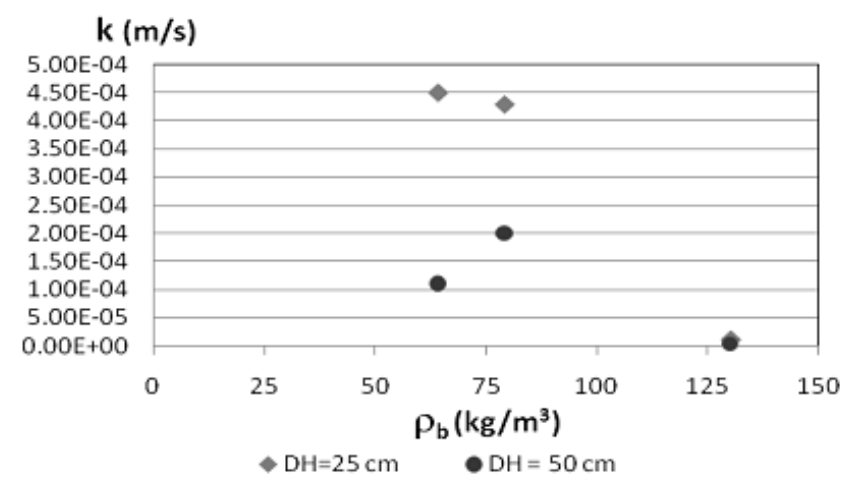

Fig. 8. Evolution of permeability to vary the density.

from the fiber. For the evaluation of this parameter a mass balance was made between the solution in the input and output from the column.

With reference to Fig. 7, the removal percentage of single contaminant is obtained as:

removal $\%=\frac{C_{\text {in }} \cdot V_{\text {in }}-C_{\text {out }}\left(V_{\text {col }}+V_{\text {out }}\right)}{C_{\text {in }} \cdot V_{\text {in }}} \cdot 100$

Where $C_{\text {in }}$ represents the initial concentration, $V_{\text {in }}$ the initial volume, $C_{\text {out }}$ the final concentration, $V_{\text {col }}$ the retained volume in the column and $V_{\text {out }}$ the output volume from the column.

\subsubsection{Kinetic of degradation}

A preliminary quantification of the capacity of removal and therefore the effectiveness of broom fiber in the treatment of heavy metals may have determined the constant of degradation $(\lambda)$ that is the parameter that allows the concentration of the contaminant in question to be linked, in this case heavy metals, with the removal rate or better with the change in concentration over time. This variation is well expressed by a first-order kinetics which is the equation used to sizing the PRB (U.S.EPA, 1998; Di Molfetta and Sethi, 2001; Bonomo, 2003):

$\frac{d C}{d t}=-\lambda \rho_{\mathrm{m}} C$

Where $\lambda$ is the parameter value which can be obtained from the experimental test, based on the values of concentrations $(C)$ of each metal collected at the exit of each column test. In particular, from the following relation:

$S=\frac{V}{A}=\frac{k i}{\lambda \rho_{\mathrm{b}}} \ln \left(\frac{C_{0}}{C}\right)$

giving the value of the thickness of the PRB, we get

$\lambda=\frac{k i}{S \rho_{\mathrm{b}}} \ln \left(\frac{C_{0}}{C}\right)$

The meaning of the symbols involved in the Eqs. (2), (3) and (4) are summarized in the list of symbols.
Table 1. Values of permeability.

\begin{tabular}{lll}
\hline & \multicolumn{2}{c}{$k(\mathrm{~m} / \mathrm{s})$} \\
& $\Delta H=25 \mathrm{~cm}$ & $\Delta H=50 \mathrm{~cm}$ \\
\hline Density $64 \mathrm{~kg} / \mathrm{m}^{3}$ & $4.50 \times 10^{-4}$ & $1.10 \times 10^{-4}$ \\
Density $79 \mathrm{~kg} / \mathrm{m}^{3}$ & $4.29 \times 10^{-4}$ & $2.00 \times 10^{-4}$ \\
Density $130 \mathrm{~kg} / \mathrm{m}^{3}$ & $1.22 \times 10^{-5}$ & $3.93 \times 10^{-6}$ \\
\hline
\end{tabular}

\section{Results and discussion}

Regarding the hydraulic conductivity measurement carried out to characterize the broom fiber, the test was repeated for three different degrees of fiber compaction within the flow cell, thus corresponding to three different densities $\left(64 \mathrm{~kg} / \mathrm{m}^{3}, 79 \mathrm{~kg} / \mathrm{m}^{3}\right.$ and $\left.130 \mathrm{~kg} / \mathrm{m}^{3}\right)$.

The values of hydraulic conductivity obtained for these three density values are shown in Table 1 .

From the analysis of the values shown in this table it can be seen that with increasing density of the fibers there is a reduction of hydraulic conductivity. This trend may be demonstrated by placing the values of conductivity obtained for each set of densities in the graph shown in Fig. 8.

Moreover, the relationships that enable the estimation of permeability $(k)$ as a function of density $(x)$ were determined. For the two considered hydraulic head, the following two expressions were obtained:

$k=-1 \times 10^{-7} x^{2}+1 \times 10^{-5} x+2 \times 10^{-5}(H=25 \mathrm{~cm})$

$k=-1 \times 10^{-7} x^{2}+3 \times 10^{-5} x-10^{-3}(H=50 \mathrm{~cm})$

The results obtained experimentally on the values of permeability assume greater importance when compared to the values of hydraulic conductivity already known from the literature for different types of soils. From this comparison, it can be said that, for the density values, with which the tests were carried out, resulting conductivities are typical of a soil constituted mainly of sand and gravel (Celico, 1986; Hamill and Bell, 1986). This highlights how, by varying the degree of compaction of the fibers, a value of permeability of the medium reagent can be obtained, in this case the broom fiber. The porosity of the broom fibers associated with a medium compaction rate, equal to 0.927 , was also measured in the laboratory.

The results from the preliminary batch tests are summarized in Table 2.

An examination of the results reported in this table shows that an increase from $P_{1}$ to $P_{2}$ in the fiber quantity, increases the heavy metals adsorption capacity. An exception to this trend is the comparison of tests 3 and 4 . This result is also evidenced by the histograms of Fig. 9, where the different situations considered for the various tests are compared. 

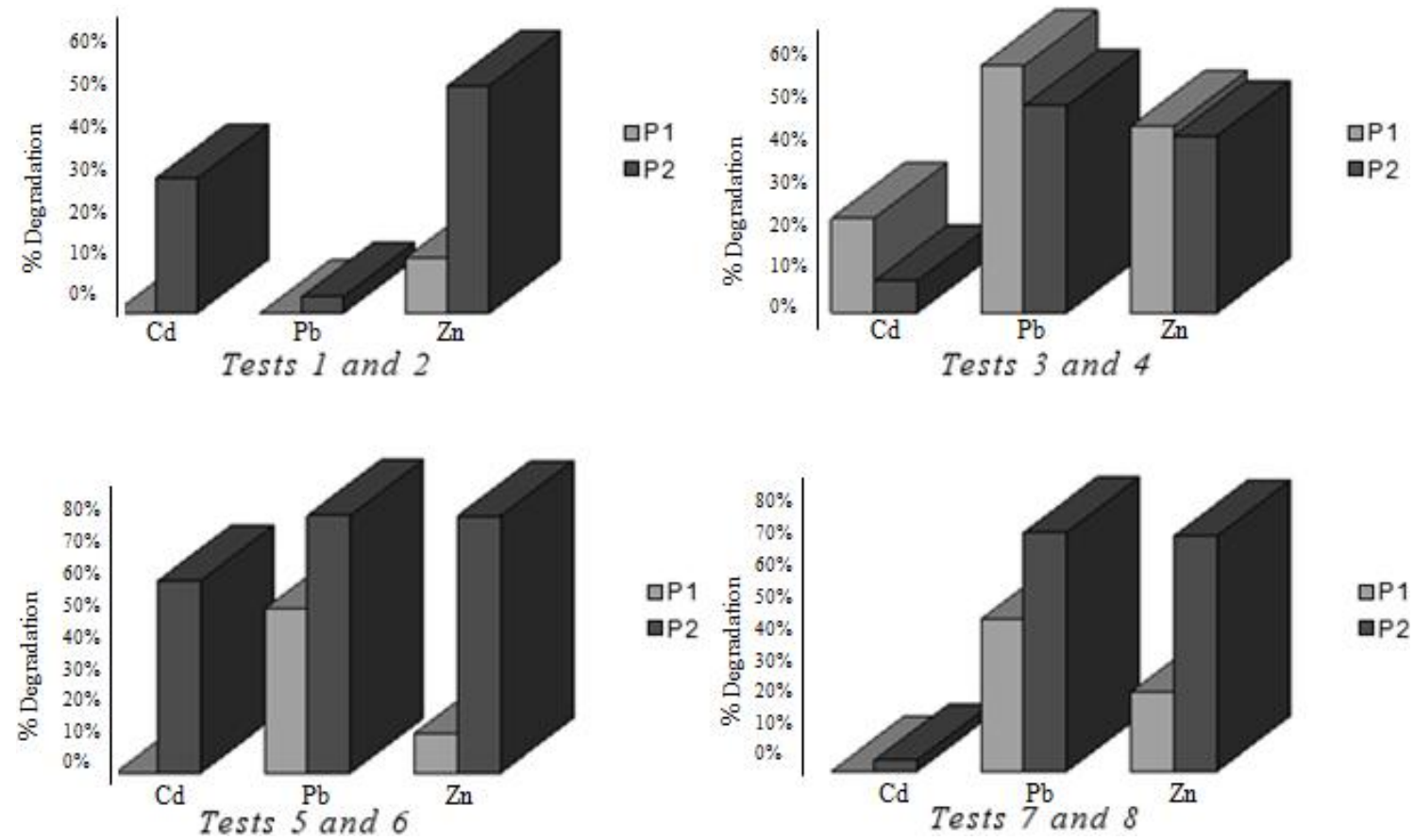

Fig. 9. Framework of union tests that show the influence of the parameter amount of fiber.

Table 2. Results of tests in batch solutions with Cadmium, Lead and Zinc.

\begin{tabular}{cccccccccccc}
\hline test & $\begin{array}{c}\mathrm{Cd}(\mu \mathrm{g} / \mathrm{l}) \\
\text { initial }\end{array}$ & $\begin{array}{c}\mathrm{Cd}(\mu \mathrm{g} / \mathrm{l}) \\
\text { final }\end{array}$ & $\begin{array}{c}\text { removal } \\
\%\end{array}$ & $\begin{array}{c}\mathrm{Pb}(\mu \mathrm{g} / \mathrm{l}) \\
\text { initial }\end{array}$ & $\begin{array}{c}\mathrm{Pb}(\mu \mathrm{g} / \mathrm{l}) \\
\text { final }\end{array}$ & $\begin{array}{c}\text { removal } \\
\%\end{array}$ & $\begin{array}{c}\mathrm{Zn}(\mu \mathrm{g} / \mathrm{l}) \\
\text { initial }\end{array}$ & $\begin{array}{c}\mathrm{Zn}(\mu \mathrm{g} / \mathrm{l}) \\
\text { final }\end{array}$ & $\begin{array}{c}\text { removal } \\
\%\end{array}$ & $\begin{array}{c}T \\
(\mathrm{~h})\end{array}$ & $\begin{array}{c}P \\
(\mathrm{~g})\end{array}$ \\
\hline 1 & 50 & $\sim 50$ & 0 & 100 & $\sim 100$ & 0 & 30000 & 28090 & 6.4 & 24 & 1 \\
2 & 50 & 42.3 & 15.4 & 100 & 98.1 & 1.9 & 30000 & 22210 & 26 & 24 & 2 \\
3 & 100 & 78.2 & 21.8 & 200 & 86.2 & 56.9 & 60000 & 34200 & 43 & 24 & 1 \\
4 & 100 & 92.6 & 7.4 & 200 & 104.7 & 47.6 & 60000 & 35550 & 40.7 & 24 & 2 \\
5 & 50 & $\sim 50$ & 0 & 100 & 55.9 & 44.1 & 30000 & 26790 & 10.7 & 48 & 1 \\
6 & 50 & 24.2 & 51.6 & 100 & 30.8 & 69.2 & 30000 & 9283 & 69 & 48 & 2 \\
7 & 100 & $\sim 100$ & 0 & 200 & 106.7 & 46.6 & 60000 & 45390 & 24.3 & 48 & 1 \\
8 & 100 & 96.6 & 3.4 & 200 & 53.3 & 73.3 & 60000 & 16680 & 72.2 & 48 & 2 \\
9 & 50 & $\sim 50$ & 0 & 100 & $\sim 100$ & 0 & 30000 & 27740 & 7.5 & 24 & 1 \\
10 & 50 & $\sim 50$ & 0 & 100 & $\sim 100$ & 0 & 30000 & 29960 & 0.1 & 48 & 2 \\
\hline
\end{tabular}

As for the contact time, this test confirms what we were expecting. At the increasing of contact time the amount of contaminant adsorbed increases and then the ratio of contaminant reduces. Again there is an outcome that does not follow the expectations, namely tests 3 and 7 . This result is shown by the histograms of Fig. 10, where they are compared with the various tests of different situations at different contact times considered.

Regarding the influence of concentration, as we expected, with increasing concentration in solution the total amount of metal cations retained by the fiber also increases, although the cadmium results in two tests is against the trend. This result is also evidenced by the histograms of Fig. 11.
As far as the fiber treated with sodium hydroxide $1 \%$ is concerned, the test results show a lack of reactivity of this fiber in relation to heavy metals, and this led us to exclude a possible influence of chemical treatment. In conclusion, the results of these batch tests were quite encouraging, in some cases removal rates above $70 \%$ were obtained, as in the case of $\mathrm{Pb}$ and $\mathrm{Zn}$ in the test No. 8 with 2 grams of fiber immersed for $48 \mathrm{~h}$ at a concentration $C_{2}$.

Regarding the column test, an objective response, in a preliminary way, of the effectiveness of treatment by filtration through the broom fibers can be obtained by analyzing the values of contaminant concentrations that the solutions presented at the end of the filtration process. Thus, knowing 

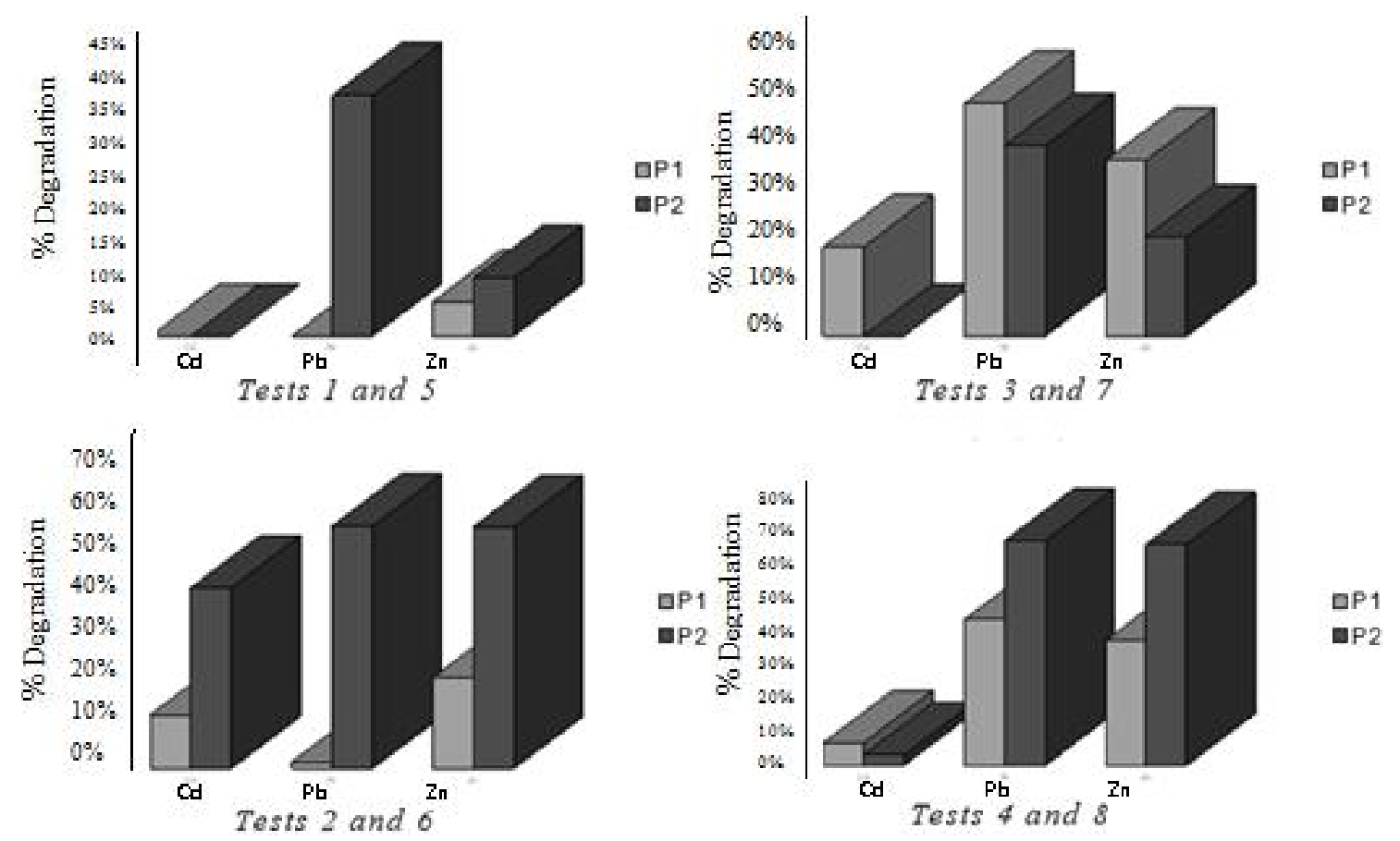

Fig. 10. Framework of union tests that show the influence of contact time parameter.
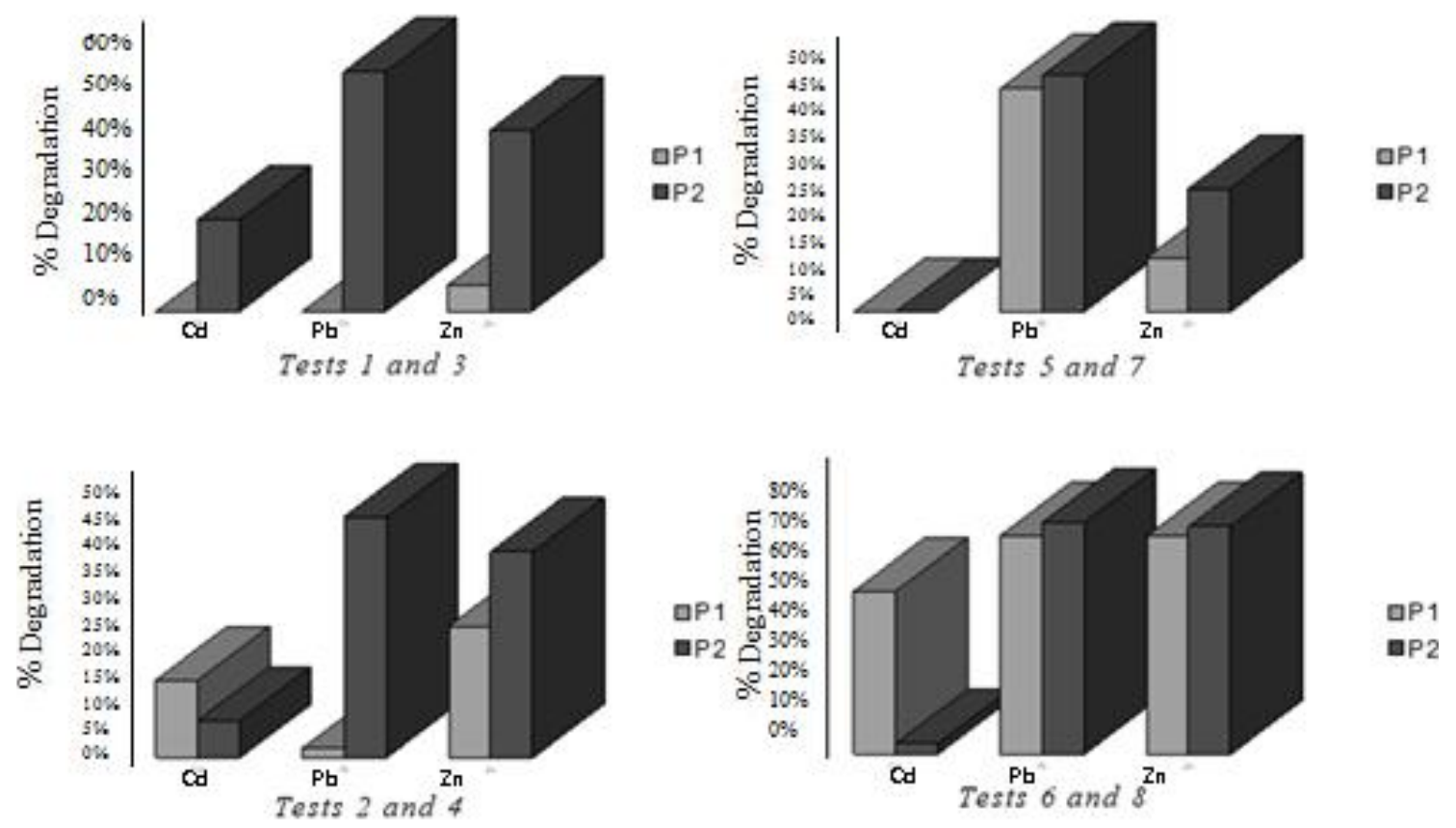

Fig. 11. Framework of union tests that show the influence of the parameter concentration.

the initial and final concentrations, the rate of removal of the contaminant can be determined and then the degree of effectiveness that the broom has on single metal assessed. The concentration values obtained are shown in Table 3 .
From the data reported in this table it can be estimated that there is a large reduction of initial concentrations. In particular, note immediately that for metals such as $\mathrm{Cu}$ and $\mathrm{Zn}$ a greater reduction is obtained compared to heavier elements such as $\mathrm{Cd}$ and $\mathrm{Pb}$. 
Table 3. Values of concentrations from the tests in the column with raw fibers and DiCoDe broom fibers.

\begin{tabular}{lccc}
\hline Element & $\begin{array}{c}\text { Initial concentration } \\
(\mathrm{g} / \mathrm{L})\end{array}$ & $\begin{array}{c}\text { Final concentration } \\
(\mathrm{g} / \mathrm{L}) \\
\text { Raw fiber }\end{array}$ & $\begin{array}{c}\text { Final concentration } \\
(\mathrm{g} / \mathrm{L}) \\
\text { DiCoDe fiber }\end{array}$ \\
\hline Copper & $1.40 \times 10^{-2}$ & $2.07 \times 10^{-5}$ & $8.24 \times 10^{-6}$ \\
Zinc & $1.18 \times 10^{-2}$ & $4.02 \times 10^{-5}$ & $6.99 \times 10^{-5}$ \\
Cadmium & $1.02 \times 10^{-5}$ & $2.58 \times 10^{-7}$ & $2.84 \times 10^{-8}$ \\
Lead & $1.07 \times 10^{-5}$ & $9.44 \times 10^{-6}$ & $3.62 \times 10^{-7}$ \\
\hline
\end{tabular}
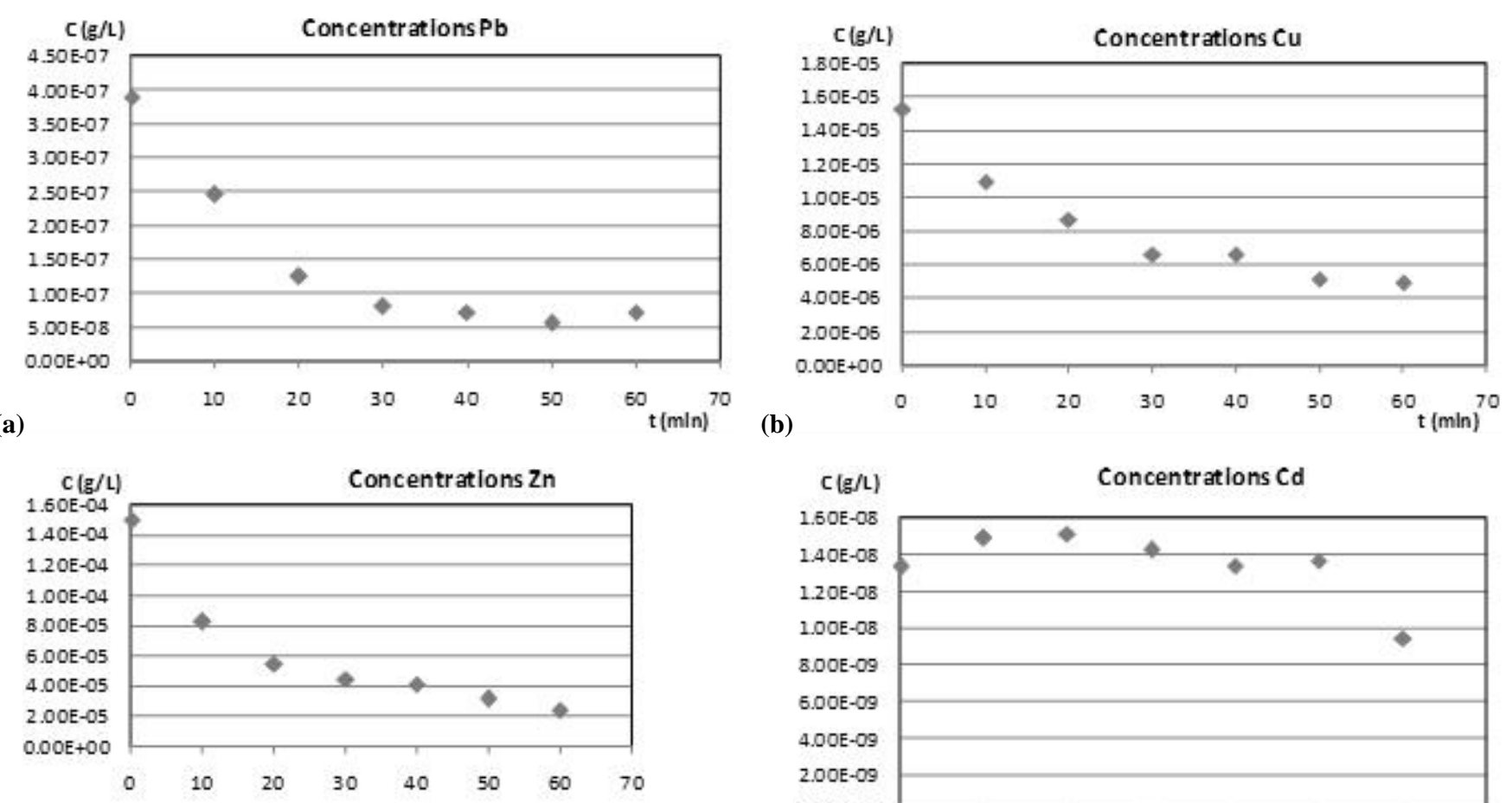

(c)

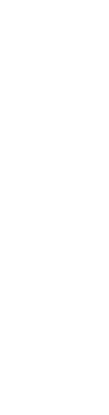

Fig. 12. Concentration-time curves derived for individual metals: (a) $\mathrm{Pb}$, (b) $\mathrm{Cu}$, (c) $\mathrm{Zn}$, (d) $\mathrm{Cd}$.

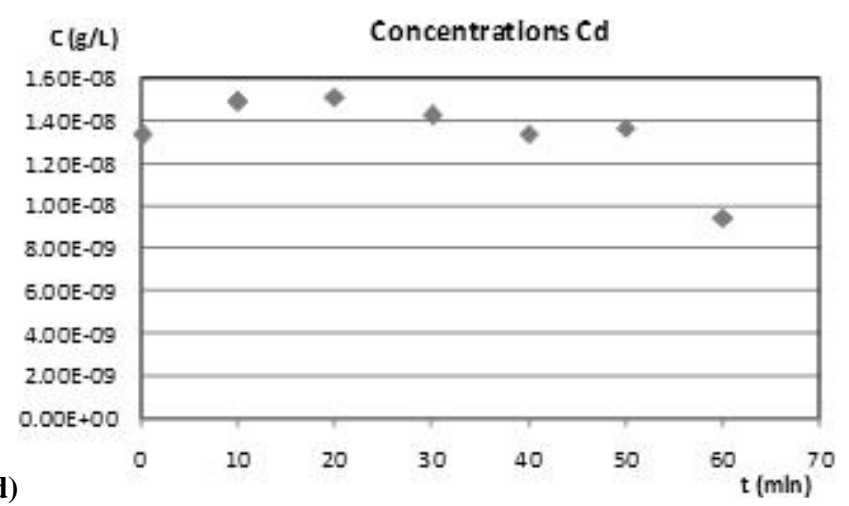

From the concentration data it can be seen how, by DiCoDe broom fibers, it is possible to have almost, in all the cases analyzed, a reduction of one order of magnitude more than the simple raw broom. By the Eq. (1), for the four considered contaminants, the following values are obtained and shown in Table 4. The values reported in this table, although they are related to a preliminary study, further corroborate the observations made previously on the basis of initial and final concentrations. In any case analyzing the data in the table it is found that with the DiCoDe broom fiber there is a slight improvement in terms of pollutant removal. This development confirms the best characteristics in terms of purification efficiency of the fiber refined. Even in the case of lead, which is the heavy metal associated with a lower rate of removal, a reduction can be obtained in concentrations under the nor- mative limit. Also considering the samples taken over time and the concentrations examined, concentration-time curves were derived for individual metals, which are shown in the graphs of Fig. 12. Looking at the graph for lead (a) it can be seen that for the first $30 \mathrm{~min}$ after exit from the column there is a rapid decrease in concentration, testifying that the fiber is carrying out an action of detention and then adsorption of the contaminant. After about $40 \mathrm{~min}$ this transient state ends and a more stable equilibrium condition is reached, as witnessed by the fact that concentrations are maintained around a mean value of $(7.025 \pm 1.027) \times 10^{-8} \mathrm{~g} / \mathrm{l}$. This steady state does not go on to infinity, but while there is capacity for adsorption on the part of the fiber. In fact, when the fiber begins to saturate, or rather when it does not hold the contaminant, the concentration should begin to rise, tending asymptotically to 
Table 4. Comparison of removal percentage of raw and DiCoDe broom fibers.

\begin{tabular}{lcc}
\hline Element & $\begin{array}{c}\text { Removal \% } \\
\text { RAW }\end{array}$ & $\begin{array}{c}\text { Removal \% } \\
\text { DiCoDe }\end{array}$ \\
\hline Lead & 36.57 & 97.35 \\
Cadmium & 97.75 & 98.01 \\
Zinc & 99.76 & 99.53 \\
Copper & 99.87 & 99.95 \\
\hline
\end{tabular}

its initial maximum value. This last aspect requires further investigation to specify carefully the behavior of the broom fiber for long times.

The graph for copper (b) shows a good efficacy of broom fiber in adsorption of that element. In particular, the decrease is characterized by a transition of about $30 \mathrm{~min}$ from the exit from the column and a subsequent equilibrium maintained until the end of sampling, carried around a value of $(5.536 \pm 0.922) \times 10^{-6} \mathrm{~g} / \mathrm{l}$.

Analyzing the graph with time concentrations of zinc (c) we can note that, even in this case, there is a decrease in concentration exit. Unlike lead, however, the transition state does not seem to finish, because the concentrations go down and then level off after 50/60 min from the exit of the column, around a mean value of $2.735 \pm 0.0001 \times 10^{-5} \mathrm{~g} / \mathrm{l}$ at which seems to reach a state of equilibrium.

Cadmium is a special case compared to the other metals studied. The development of cadmium with time (d) shows, in fact, how the values of the output concentrations remain virtually constant around the value of $(1.411 \pm 0.077) \times 10^{-8} \mathrm{~g} / \mathrm{l}$. This trend is justified by the fact that the kinetics of adsorption of cadmium in the column containing DiCoDe broom fiber is much faster than the other three cases analyzed, because the concentration values that are obtained can be considered as already relative to steady state of equilibrium. In this case, therefore, it can be said that the transition phase, characterized by a progressive decrease in concentrations with time, has already taken place inside the column during the filling phase of the column by solution with $\mathrm{Cd}$. This phase has a duration of $90 \mathrm{~min}$.

Moreover, applying the relation (4) it was considered that the thickness of the barrier in the direction of flow can be represented by the height of the column, the bulk density is set equal to the density of broom fibers in the column, the conductivity has the value derived from the permeability test specially realized, the value of the gradient is associated with the value of the porosity obtained with an intermediate rate of compaction of fibers in the column and associated with the value of a filtration speed of about $10 \mathrm{~m} /$ day. Finally, the values of the concentrations are respectively the initial concentration $C_{0}$ leaked from the column, while $C$ expresses the value of concentration that is obtained at the exit of column in the various times considered. More specifically the value of the permeability, associated with an intermediate degree of compaction of the fibers, comes from the permeability tests carried out previously and it is $2 \times 10^{-4} \mathrm{~m} / \mathrm{s}$.

Whereas in all the tests the filtration speed is about $10 \mathrm{~m} /$ day, knowing the inner section of the column a gradient of 0.34 was obtained. The other values that appear in the previous equation are $S=0.6 \mathrm{~m}$ and $\rho_{\mathrm{b}}=79 \mathrm{~kg} / \mathrm{m}^{3}$. Using these quantities and taking into account the values of the initial concentration and various concentrations over time, for each metal the average values of $\lambda$ reported in Table 5 were obtained. Analyzing the values of the constant degradation identified for each metal, it can be seen that, in principle, the broom has a rate of removal of the order of $10^{-1} \mathrm{~m}^{3} /(\mathrm{kg}$ day). More specifically, there is a higher $\lambda$ and a greater rate of decrease of the contaminant concentration in the case of copper, while the lowest is found in the case of lead. Examination of Table 5 shows that the lead needs more time to be removed, unlike copper, which has a higher value of $\lambda$ and for this needs less time for its removal.

\section{Conclusions}

The aim of the present work is the characterization of broom fiber for its possible use as a means of reagent in the remediation of soils contaminated by heavy metals. For this purpose a hydraulic characterization of the mass of broom fiber was carried out to determine its permeability and porosity. Then the adsorption capacity of broom fiber was tested considering some heavy metals, by performing tests in batches with solutions of cadmium, lead and zinc. Based on the encouraging results obtained, the tests were conducted in column using contaminating elements such as $\mathrm{Cd}, \mathrm{Pb}, \mathrm{Zn}$ and $\mathrm{Cu}$. From the permeability test performed for three different degrees of compaction (and therefore different densities) and with two different values of the hydraulic head an appropriate set of general and preliminary equations were obtained, which allow the definition of the degree of compaction that the fibers must have to obtain a fixed value of permeability of the medium depending on the hydraulic head of the aquifer that needs to be reclaimed. These equations are found from experimental interpolation data and therefore are valid in the field of bulk density investigated. The measures of porosity provided a very high porosity of $92.7 \%$ and this is the evidence which shows that the broom fibers have a high percentage of voids and therefore of sites for containment/treatment of contaminants. For a more accurate characterization of the broom reagent the same test was carried out in the column on both raw broom fiber (broom with shoots simply washed and dried) and on DiCoDe broom fiber (shoots treated by chemical-physical process). Analyzing the data of the concentrations obtained after the filtration tests it was observed that passing from the raw broom fiber to DiCoDe broom fiber, there was a good increase in the reduction 
Table 5. Average decay constants for each metal.

\begin{tabular}{ccccc}
\hline$t$ (min) & $\begin{array}{c}\lambda \\
{\left[\mathrm{m}^{3} /(\mathrm{kg} \text { day })\right]} \\
\text { Lead }\end{array}$ & $\begin{array}{c}\lambda \\
{\left[\mathrm{m}^{3} /(\mathrm{kg} \text { day })\right]} \\
\text { Zinc }\end{array}$ & $\begin{array}{c}\lambda \\
{\left[\mathrm{m}^{3} /(\mathrm{kg} \text { day })\right]} \\
\text { Copper }\end{array}$ & $\begin{array}{c}\lambda \\
{\left[\mathrm{m}^{3} /(\mathrm{kg} \text { day })\right.} \\
\text { Cadmium }\end{array}$ \\
\hline 0 & 0.410 & 0.541 & 0.845 & 0.823 \\
10 & 0.468 & 0.616 & 0.887 & 0.809 \\
20 & 0.552 & 0.667 & 0.916 & 0.807 \\
30 & 0.606 & 0.693 & 0.950 & 0.815 \\
40 & 0.620 & 0.702 & 0.950 & 0.823 \\
50 & 0.651 & 0.737 & 0.981 & 0.820 \\
60 & 0.619 & 0.769 & 0.986 & 0.866 \\
& \multicolumn{4}{c}{ Mean value } \\
\end{tabular}

of the concentration and therefore greater efficiency in terms of pollutant removal. From the obtained results, it can be affirmed that with the DiCoDe broom fibers there was a higher reduction in the concentrations of contaminants compared a simple raw broom.

These results are further supported by analyzing the removal percentage, which confirms the best characteristics in terms of purification efficiency of the refined fiber.

In all cases analyzed there is, in fact, a high removal efficiency with a significant reduction of the concentration of single pollutants, as witnessed by the fact that the removal percentage obtained from the experimental tests is very close to $100 \%$.

Having found the best purifying capacity of DiCoDe broom fibers, it was decided to further characterize the broom by means of the column test, with various sampling to determine the behavior of the fiber with time and the constant degradation $\lambda$. From the results obtained it was possible to predict approximately, owing to different chemical and physical conditions in the groundwater, the behavior of the fiber in adsorption of each of the contaminants analyzed. Analyzing the data obtained it was found that, using the broom for the remediation of groundwater contaminated by heavy metals, lead is a more difficult pollutant to remove by natural fibers, because it needs more culling time, unlike copper, which is characterized by a higher value of $\lambda$ and therefore needs less time to degrade. In every case knowing $\lambda$ allows one to proceed to a preliminary calculation of the volume and amount of reactive media necessary to achieve a target of removal and concentration reduction of contaminants with an appropriately fixed target value.

The results shown in the present paper induce one to retain the broom fibers good material to use for PRB. In fact, they contain a quantity of total lignin comparable to that of other natural fibers, so it proves very effective to retain heavy metals, and it results very competitive, because it offers considerable economic advantages owing to its very low cost. How- ever, other investigations are required for a better knowledge of the characteristics of the broom fibers and of their behavior, particularly over long times, as material for the PRB.

\section{Appendix A}

\section{List of symbols}

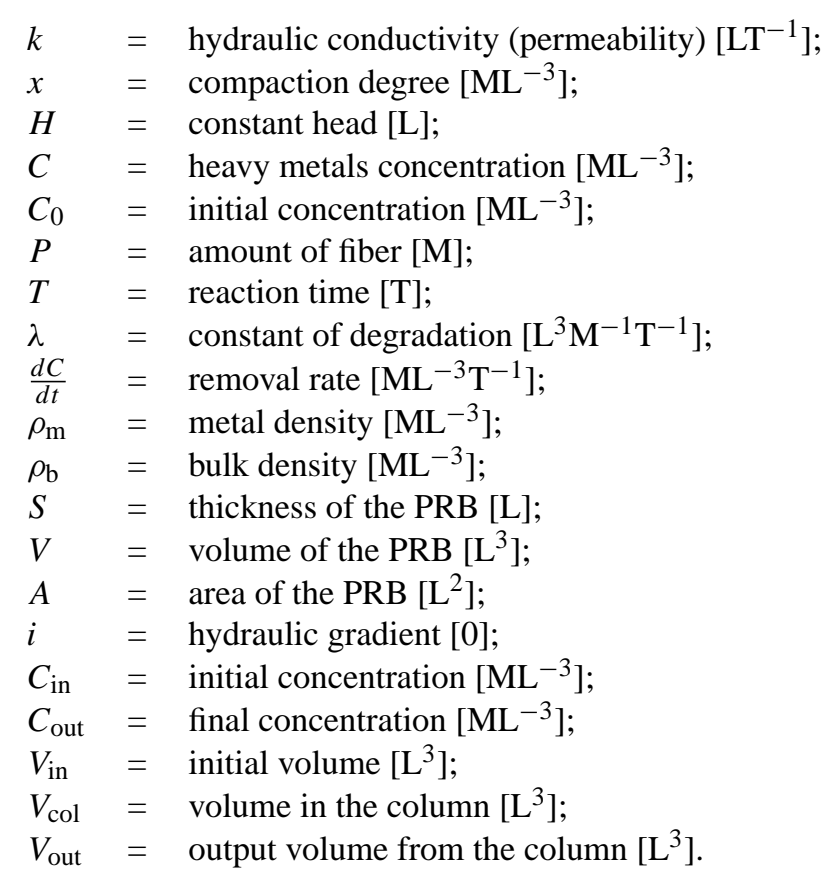


Edited by: S. De Bartolo

\section{References}

Angelini, L. G., Lazzeri, A., Levita, G., Fontanelli, D., and Bozzi, C.: Ramie (Boehmeria nivea (L.) Gaud.) and Spanish Broom (Spartium junceum L.) fibres for composite materials: agronomical aspects, morphology and mechanical properties, Industrial Crops and Products, 11, 145-161, 2000.

Bonomo, L.: 57th Corso di aggiornamento in Ingegneria SanitariaAmbientale. Siti Contaminati: Tecnologie di risanamento, Dipartimento di Ingegneria Idraulica, Ambientale e del Rilevamento Sezione Ambientale - Politecnico di Milano, 2003.

Celico, P.: Prospezioni Idrogeologiche, vol. 1, Liguori Ed., Napoli, 1986.

Chidichimo, G., Gabriele, B., Salerno, G., Alampi, C., Cerchiara, T., and Vetere, M.: Processo chimico-fisico per la produzione di fibre vegetali, Brevetto dell'Università della Calabria (No.: CZ2006A00006), 2006.

Di Molfetta, A. and Sethi, R.: Metodologie di bonifica di siti contaminati, Siti contaminati, 1, 16-24, 2000.

Di Molfetta, A. and Sethi, R.: Criteri di progettazione di barriere permeabili reattive a ferro zero valente, Dipartimento di Georisorse e Territorio, Politecnico di Torino, 2001.

Gabriele, B., Cerchiara, T., Salerno, G., Chidichimo, G., Vetere, M. V., Alampi, C., Gallucci, M. C., Conidi, C., and Cassano, A.: A new physical-chemical process for the efficient production of cellulose fibers from Spanish broom (Spartium junceum L.), Bioresource Technology, 101, 724-729, 2009.

Gillham, R. W. and O'Hannesin, S. F.: Enhanced degradation of halogenated aliphatics by zerovalent iron, Ground Water, 32, 958-967, 1994.

Hamill, L. and Bell, F. G.: Ground water resource development, Butterworth Heinemann Ltd. Oxford, 1986.

Jun, D., Yongsheng, Z., Weihong, Z., and Mei, H.: Laboratory study on sequenced permeable reactive barrier remediation for landfill leachate-contaminated groundwater, J. Hazardous Materials, 161, 224-230, 2009.
Lee, B. G. and Rowell, R. M.: Removal of heavy metal ions from aqueous solutions using lignocellulosic fibers, J. Natural Fibers, 1, 97-108, 2004.

Ministero dell'Ambiente: Decreto Legislativo 3 aprile 2006, n. 152: Norme in materia ambiente. SO alla GU della Repubblica Italiana n. 88 del 14 Aprile 2006 - Suppl. Ordinario n. 9, 2006.

Mohanty, A. K., Misra, M., and Hinrichsen, G.: Biofibres, biodegradable polymers and biocomposites, Macromol. Mater. s.1.: Wiley-VCH, 276/277, 1-24, 2000.

Orth, S. and Gillham, R.: Dechlorination of Trichloroethene in Aqueous Solution Using $\mathrm{Fe}^{0}$, Environ. Sci. Technol., 30, 66-77, 1996.

Orth, F., Dauda, T., and Mckenzie, D. E.: Reductive Dechlorination of DNAPL Trichloroethylene by Zero-Valent Iron. Pract. Periodical of Haz., Toxic, and Radioactive Waste Mgmt., 2, 123-128, 1998.

Parbs, A. and Birke, V.: State-of-the-art report and inventory on already demonstrated innovative remediation technologies, EURODEMO (European Co-ordination Action for Demonstration of Efficent Soil and Groundwater Remediation), University of Lneburg (ULB, formerly University of Applied Sciences NE Lower Saxony, FHNON), 2005.

Powell, R. M., Puls, R. W., Blowes, D. W., and Vogan, J. L.: Permeable Reactive Barrier Technologies for Contaminant Remediation, U.S. Environmental Protection Agency, US Office of Research and Development, US Office of Solid Waste and Emergency Response, EPA/600/R-98/125, 1998.

Troisi, S., Fallico, C., Straface, S., and Mazzuca, L.: Biodreni per la bonifica di siti contaminati realizzati con fibre naturali liberiane ad elevato sviluppo superficiale. Brevetto dell'Università della Calabria (n.: CS2008A00018), 2008.

U.S.EPA: Permeable reactive barrier. Technologies for contaminant remediation, edited by: Powell, R. M. and Puls, R. W., Subsurface Protection and Remediation Division National Risk Management Research Laboratory Ada, Oklahoma 74820, September, 1998.

Zolla, V., Sethi, R., and Di Molfetta, A.: Performance assessment and monitoring of a permeable reactive barrier for the remediation of a contaminated site, Am. J. Environ. Sci., 3, 158-165, 2007. 